\title{
Cardiorenal Syndrome
}

National Cancer Institute

\section{Source}

National Cancer Institute. Cardiorenal Syndrome. NCI Thesaurus. Code C123225.

A disorder of the heart and kidneys in which dysfunction of one of the organs induces dysfunction of the other organ. 